\title{
ON $C^{m}$-BOUNDING SETS
}

\section{PETER BISTRÖM, STEN BJON and MIKAEL LINDSTRÖM}

(Received 14 May 1991)

Communicated by P. G. Dodds

\begin{abstract}
Let $E$ be a quasi-complete locally convex space and $A$ a subset of $E$. It is shown that if every real-valued $C^{\infty}$-function in the weak topology of $E$ is bounded on $A$, then $A$ is relatively weakly compact. Furthermore, if all real-valued $C^{\infty}$-functions on $E$ are bounded on $A$, then $A$ is relatively compact in the associated semi-weak topology of $E$.
\end{abstract}

1991 Mathematics subject classification (Amer. Math. Soc.): 46 A 99, 58 C 25.

Keywords and phrases: $C^{m}$-bounding set, relatively compact set, semi-weak topology, interchangeable double limit property.

The purpose of this paper is to examine the $C^{m}$-bounding sets ( $m=0$, $1, \ldots, \infty)$ in a real Hausdorff locally convex space (lcs), with emphasis on their connection with relatively compact sets in various topologies. We say that a subset $A$ of a lcs $E$ is $C^{m}$-bounding if every $f \in C^{m}(E)$ is bounded on $A$. Here, $C^{m}(E)$ denotes the set of all real-valued $C^{m}$-functions on $E$ in the sense of Lloyd [13], whose definition of differentiability coincides with that of Fréchet on Banach spaces. $C^{\infty}$-bounding sets have recently been investigated by Kriegl and $\mathrm{Nel}$ in [11].

Since $E^{\prime} \subset C^{m}(E) \subset C(E)$ for every lcs $E$, the class of $C^{m}$-bounding sets is somewhere between the classes of bounded and relatively compact sets. The $C^{0}$-bounding subsets of a lcs $E$ are relatively compact if $E$ is quasicomplete or metrizable or, by [10], if $E$ is realcompact. Moreover, Valdivia proves in [15] that every set, which is $C^{0}$-bounding in the weak topology of a

(C) 1993 Australian Mathematical Society 0263-6115/93 \$A2.00+0.00 
quasi-complete lcs, is relatively weakly compact. We obtain a generalization of this result to $C^{\infty}$-bounding sets.

For the study of $C^{\infty}$-bounding sets in Banach spaces it is convenient to consider the finest semi-weak topology compatible with the duality $\left\langle E, E^{\prime}\right\rangle$ [4]. This locally convex topology, which we denote by $E_{s w}$, is strictly finer than the associated Schwartz topology and the weak topology on $E$. Our main theorem states that every $C^{\infty}$-bounding set in a Banach space $E$ is relatively compact in $E_{s w}$. This should be compared with a deep result due to Bourgain and Diestel [3], by which the limited sets in a Banach space not containing $l_{1}$ are relatively weakly compact. Since the $C^{\infty}$-bounding sets form a proper subclass of the limited sets, it follows from the theory of limited sets that the $C^{\infty}$-bounding subsets of various Banach spaces are relatively compact. However, using the associated semi-weak structure, we obtain the same results in a general way. Further, we show that the classes of $C^{\infty}$-bounding and relatively compact sets coincide in Lindelöf as well as in quasi-complete smooth lcs.

The following concept guarantees a rich supply of functions in $C^{m}(E)$. A lcs $E$ is $C^{m}$-smooth if there, for every point $a \in E$ and open subset $V$ containing $a$, exists a function $f \in C^{m}(E)$ with $f(a)>0$ and $f(x)=$ 0 for $x \notin V$. The class of $C^{m}$-smooth spaces is closed under formation of subspaces and arbitrary products [13]. All Schwartz spaces [13] and the Banach spaces $c_{0}(\Gamma), l_{2 n}(\Gamma)$ and $L_{2 n}(\mu)$ are well-known examples of $C^{m}$. smooth spaces.

Proposition 1. Let $E$ be a $C^{m}$-smooth lcs. Then every $C^{m}$-bounding subset of $E$ is precompact and hence also relatively compact if, in addition, $E$ is quasi-complete.

Proof. Assume that $A \subset E$ is not precompact. Then there exist a sequence $\left(x_{n}\right)$ in $A$ and a zero-neighbourhood $U$ in $E$ with $x_{n}-x_{m} \notin U$ for $n \neq m$. Let $V$ be an open set in $E$ with $0 \in V$ and $V-V \subset U$. By the $C^{m}$-smoothness of $E$ there is for each $n$ a function $f_{n} \in C^{m}(E)$ such that $f_{n}\left(x_{n}\right)=n$ and $f_{n}(x)=0$ for $x \notin x_{n}+V$. Let $f(x)=\sum_{n=1}^{\infty} f_{n}(x)$. By construction the sum $f$ is locally finite and thus of class $C^{m}$. Since $f\left(x_{n}\right)=n$ for each $n$, the set $A$ cannot be $C^{m}$-bounding.

Let $E_{m}$ be the set $E$ endowed with the weakest topology making all $f \in$ $C^{m}(E)$ continuous. Then $E_{m}$ is a completely regular Hausdorff topological space. Clearly $E=E_{0}$ and $E$ is $C^{m}$-smooth if and only if $E=E_{m}$.

Proposition 2. Let $E$ be a lcs and suppose that there is a sequence in 
$C^{m}(E)$ which separates the points of $E$. Then every $C^{m}$-bounding subset $A$ of $E$ is relatively compact in $E_{m}$.

Proof. Let $A \subset E$ be $C^{m}$-bounding. The set $f(A) \subset R$ is relatively compact for every $f \in C^{m}(E)$, thus $A$ must be relatively compact in $R^{C^{m}(E)}$, by Tychonoff's theorem. Since $C^{m}(E)$ admits a sequence which separates the points of $E$, it follows from corollary 4 in [2] that $E$ equals Hom $C^{m}(E)$, the set of all real-valued non-zero homomorphisms on $C^{m}(E)$. Now Hom $C^{m}(E)$ is a closed subset of $R^{C^{m}(E)}$ and therefore $A$ is relatively compact in $E_{m}$.

The dual of a lcs $E$ is $\sigma\left(E^{\prime}, E\right)$-separable if and only if $E^{\prime}$ admits a sequence which separates the points of $E:$ Let $\left(l_{n}\right)$ be such a sequence and let $l \in E^{\prime}$ be arbitrary. For each finite-dimensional subspace $F$ of $E$, there is an $l_{F}$ in the linear span of $\left(l_{n}\right)$ with $l_{F}(x)=l(x)$ for each $x \in F$. Since the net $\left(l_{F}\right)$ converges to $l$ in $\sigma\left(E^{\prime}, E\right)$, we conclude that $E^{\prime}$ is $\sigma\left(E^{\prime}, E\right)$-separable. The converse statement is trivial. Now, $\sigma\left(E, E^{\prime}\right)$ being $C^{\infty}$-smooth, we obtain from Proposition 2 the following

CoRollary 3. If $E$ is a lcs with $\sigma\left(E^{\prime}, E\right)$-separable dual, then every $C^{\infty}$. bounding subset of $\left(E, \sigma\left(E, E^{\prime}\right)\right)$ is relatively $\sigma\left(E, E^{\prime}\right)$-compact.

A subset $A$ of a lcs $E$ is called limited [12], if each equicontinuous $\sigma\left(E^{\prime}, E\right)$-null sequence $\left(l_{n}\right)$ in $E^{\prime}$ converges to zero uniformly on $A$. Every equicontinuous $\sigma\left(E^{\prime}, E\right)$-null sequence $\left(l_{n}\right)$ defines a linear and continuous mapping $T: E \rightarrow c_{0}, x \mapsto\left(l_{n}(x)\right)_{n \in N}$. Hence, by [1, p. 161], $A$ is limited, if $T(A)$ is relatively compact in $c_{0}$ for all $T \in L\left(E, c_{0}\right)$. Thus, by Proposition 1 , every $C^{\infty}$-bounding set in $E$ is limited. However, the converse does not hold in general. According to [12] all precompact sets are limited. Therefore the limited sets in $\left(E, \sigma\left(E, E^{\prime}\right)\right)$ are exactly the bounded ones for every lcs $E$. If $E$ is a non-semi-reflexive lcs with $\sigma\left(E^{\prime}, E\right)$-separable dual (e.g. $l^{\infty}$ or $c_{0}$ ), the corollary above ensures the existence of limited sets in lcs which are not $C^{\infty}$-bounding.

There exist even limited non- $C^{\infty}$-bounding sets in Banach spaces. Indeed, by Phillips' lemma [1, p. 233], a sequence $\left(l_{n}\right)$ in $\left(l^{\infty}\right)^{\prime}$ converges uniformly to zero on the closed unit ball $B_{c_{0}}$ of $c_{0}$, if $\left(l_{n}\right)$ converges to zero in $\sigma\left(\left(l^{\infty}\right)^{\prime}, l^{\infty}\right)$. This means that the non-compact set $B_{c_{0}} \subset l^{\infty}$ is limited in $l^{\infty}$. Assume that $B_{c_{0}} \subset l^{\infty}$ is also $C^{\infty}$-bounding in $l^{\infty}$. Since $B_{c_{0}}$ is closed in $\sigma\left(l^{\infty},\left(l^{\infty}\right)^{\prime}\right)$, Corollary 3 implies that $B_{c_{0}}$ is $\sigma\left(l^{\infty},\left(l^{\infty}\right)^{\prime}\right)$-compact. But, since $\sigma\left(l^{\infty},\left(l^{\infty}\right)^{\prime}\right)$ and $\sigma\left(c_{0}, l_{1}\right)$ coincide on $B_{c_{0}}$, we conclude that $B_{c_{0}}$ is 
$\sigma\left(c_{0}, l_{1}\right)$-compact, which is a contradiction. Hence $B_{c_{0}} \subset l^{\infty}$ is a limited non- $C^{\infty}$-bounding set in $l^{\infty}$.

If we leave the use of Corollary 3 out of account, the proof of the next theorem has much in common with the corresponding proof in the case $m=$ 0 as carried out in [8, p. 24]. But since the result is important and will be used in the sequel, we include a full detailed proof for the sake of completeness.

Let $\left\langle E, E^{\prime}\right\rangle$ be a dual pair. We say that two sets $A \subset E$ and $M \subset E^{\prime}$ have the interchangeable double limit property, if for every sequence $\left(x_{m}\right)$ in $A$ and every sequence $\left(l_{n}\right)$ in $M$ the double $\operatorname{limits}_{m} \lim _{n}\left\langle x_{m}, l_{n}\right\rangle$ and $\lim _{n} \lim _{m}\left\langle x_{m}, l_{n}\right\rangle$ are equal if they exist. The Eberlein-Grothendieck theorem [8, p. 15] states that a subset $A$ of a lcs $E$ is bounded and has the interchangeable double limit property with all equicontinuous subsets of $E^{\prime}$, if $A$ is relatively $\sigma\left(E, E^{\prime}\right)$-compact. The converse implication is true if $E$ is quasi-complete.

THEOREM 4. Let $E$ be a quasi-complete lcs. Then every $C^{\infty}$-bounding subset $A$ of $\left(E, \sigma\left(E, E^{\prime}\right)\right)$ is relatively $\sigma\left(E, E^{\prime}\right)$-compact.

Proof. Take an equicontinuous sequence $\left(l_{n}\right)$ in $E^{\prime}$ and a sequence $\left(x_{m}\right)$ in $A$ such that the corresponding double limits exist. Let $F$ be the $\sigma\left(E^{\prime}, E\right)$ closed linear span of $\left(l_{n}\right)$. Then $F=\left(E / F^{\circ}\right)^{\prime}$ is $\sigma\left(F, E / F^{\circ}\right)$-separable and the canonical linear mapping $\pi:\left(E, \sigma\left(E, E^{\prime}\right)\right) \rightarrow\left(E / F^{\circ}, \sigma\left(E / F^{\circ}, F\right)\right)$ is continuous. Hence $\pi(A)$ is relatively $\sigma\left(E / F^{\circ}, F\right)$ )-compact by Corollary 3 . The sequence $\left(l_{n}\right)$ is equicontinuous in $F=\left(E / F^{\circ}\right)^{\prime}$, when $E / F^{\circ}$ has the quotient topology of $E$. By the interchangeable double limit property for the duality $\left\langle E / F^{\circ}, F\right\rangle$,

$$
\begin{aligned}
& \lim _{m} \lim _{n}\left\langle x_{m}, l_{n}\right\rangle_{E, E^{\prime}}=\lim _{m} \lim _{n}\left\langle\pi\left(x_{m}\right), l_{n}\right\rangle_{E / F^{\circ}, F} \\
= & \lim _{n} \lim _{m}\left\langle\pi\left(x_{m}\right), l_{n}\right\rangle_{E / F^{\circ}, F}=\lim _{n} \lim _{m}\left\langle x_{m}, l_{n}\right\rangle_{E, E^{\prime}} .
\end{aligned}
$$

Since $E$ is quasi-complete, $A$ is relatively $\sigma\left(E, E^{\prime}\right)$-compact.

The theorem above extends a corresponding result of Valdivia for $C^{0}$ bounding sets to $C^{\infty}$-bounding ones [15]. Similar results for limited sets have been obtained under much more restrictive conditions: In [3] Bourgain and Diestel show that in Banach spaces containing no copy of $l_{1}$ all limited sets are relatively weakly compact.

Since $l_{1}(\Gamma)$ and $\sigma\left(l_{1}(\Gamma), l^{\infty}(\Gamma)\right)$ have the same compact sets, we notice that the $C^{\infty}$-bounding subsets of $l_{1}(\Gamma)$ are relatively compact also for uncountable sets $\Gamma$. 
Corollary 5. Let $E$ be a Banach space. If $E^{\prime}$ admits a sequence that separates the points of $E$, then every $C^{m}$-bounding subset $A$ of $E$ is metrizable and relatively sequentially compact with respect to $E_{m}$.

Proof. According to Theorem 4, the set $\bar{A}^{\sigma}$ is compact in $\sigma\left(E, E^{\prime}\right)$. Since $E^{\prime}$ contains a countable set that separates the points of $E$, the set $\bar{A}^{\sigma}$ is metrizable in $\sigma\left(E, E^{\prime}\right)$, by theorem 10.11 in [1]. The identity mapping id $:\left(\bar{A}^{E_{m}}, E_{m}\right) \rightarrow\left(\bar{A}^{E_{m}}, \sigma\left(E, E^{\prime}\right)\right)$ is continuous. By Proposition $2, \bar{A}^{E_{m}}$ is compact in $E_{m}$, and therefore $i d$ is a homeomorphism. Thus $\sigma\left(E, E^{\prime}\right)$ and $E_{m}$ coincide on $\bar{A}^{E_{m}}$ and the corollary follows.

Proposition 6. Let $E$ be a lcs such that every $C^{m}$-bounding subset of $E$ is relatively compact in $E$. Then $E$ and $E_{m}$ have the same convergent sequences and compact sets.

Proof. Since the functions in $C^{m}(E)$ are continuous with respect to $E_{m}$, every compact set $A$ in $E_{m}$ is $C^{m}$-bounding in $E$. Therefore $A$ is compact in $E$. Further, let $x_{n} \rightarrow x$ in $E_{m}$. Then $B=\left\{x_{n}: n \in N\right\} \cup\{x\}$ is compact in $E_{m}$ and hence also in $E$. The topologies of $E$ and $E_{m}$ coincide on $B$ and consequently $x_{n} \rightarrow x$ in $E$.

Remark. Let $E$ be a Banach space and assume that $l^{\infty}$ and $\left(l^{\infty}\right)_{m}$ define the same convergent sequences. Then it can easily be proved using Corollary 5 and proposition 4 in [6] that every $C^{m}$-bounding subset of $E$ is relatively compact in $E$.

The semi-weak lcs are exactly those which are isomorphic to subspaces of some product $c_{0}{ }^{I}$ [4]. Every lcs $E$ defines a semi-weak space $E_{s w}$ which is obtained by endowing the set $E$ with the topology of uniform convergence on the $\sigma\left(E^{\prime}, E\right)$-closed absolutely convex hulls of all sets of the form $\left\{l_{n}\right.$ : $n \in N\}$, where $\left(l_{n}\right)$ is an equicontinuous sequence in $E^{\prime}$ which converges to zero in $\sigma\left(E^{\prime}, E\right)$. The topology of $E_{s w}$ is the finest semi-weak topology, which is coarser than the topology of $E$ [4]. Note that $E^{\prime}=\left(E_{s w}\right)^{\prime}$ and that the topology of $E_{\infty}$ is finer than $E_{s w}$, since $E_{s w}$ is $C^{\infty}$-smooth.

THEOREM 7. If $E$ is a quasi-complete lcs, then every $C^{\infty}$-bounding subset $A$ of $E$ is relatively compact in $E_{s w}$.

Proof. Since $A$ is $C^{\infty}$-bounding in $E$, Theorem 4 yields that $\bar{A}^{\sigma}$ is weakly compact and therefore complete in $\sigma\left(E, E^{\prime}\right)$. Now $E^{\prime}=\left(E_{s w}\right)^{\prime}$ so, by theorem 3.24 in [10], the set $\bar{A}^{\sigma}$ is complete in $E_{s w}$. Since $E_{s w}$ 
is $C^{\infty}$-smooth, $A$ is precompact in $E_{s w}$, by Proposition 1. The zeroneighbourhoods in $E_{s w}$ are $\sigma\left(E, E^{\prime}\right)$-closed, so we conclude that $\bar{A}^{\sigma}$ is precompact and hence also compact in $E_{s w}$.

Theorem 7, our main result, lifts the relative compactness of the $C^{\infty}$ bounding sets on $E$ from the weak topology, as in Theorem 4, to the finer semi-weak topology. In fact, the lifting works for any $C^{\infty}$-smooth locally convex topology compatible with the duality $\left\langle E, E^{\prime}\right\rangle$. The semi-weak space $E_{s w}$ is in general strictly finer than the associated Schwartz space $E_{s z}$ (by proposition 5.10 in [4] a Banach space $E$ is finite-dimensional if $E_{s w}=E_{s z}$ ). Furthermore, using the associated semi-weak topology we obtain in a unified manner various results, proved for limited sets by different methods.

Corollary 8. Every $C^{\infty}$-bounding subset of a Banach space $E$ is relatively sequentially compact in $E_{\text {sw }}$.

Proof. According to Theorem 7 we have to show that every relatively compact set $A$ in $E_{s w}$ is relatively sequentially compact in $E_{s w}$. Now the $E_{s w}$-closure of $A$ is compact in $E_{s w}$ and, by the Eberlein-Šmulian theorem, $A$ is relatively sequentially $\sigma\left(E, E^{\prime}\right)$-compact. By compactness, $\sigma\left(E, E^{\prime}\right)$ and $E_{s w}$ coincide on $\bar{A}^{E_{s w}}$, and therefore $A$ is relatively sequentially compact in $E_{s w}$.

Let $E$ be a Banach space and $\mu\left(E^{\prime}, E\right)$ the Mackey topology on $E^{\prime}$. The topology $\mu\left(E^{\prime}, E\right)_{s w}$ is the topology of uniform convergence on the closed absolutely convex hulls of all sequences converging to zero in $\sigma\left(E, E^{\prime}\right)$.

LEMMA 9. Let $E$ be a Banach space that contains no subspace isomorphic to $l_{1}$. Then $\beta\left(E^{\prime}, E\right), \mu\left(E^{\prime}, E\right)$ and $\mu\left(E^{\prime}, E\right)_{\text {sw }}$ have the same convergent sequences.

Proof. Suppose that $l_{n} \rightarrow 0$ in $\mu\left(E^{\prime}, E\right)_{s w}$ but not in $\beta\left(E^{\prime}, E\right)$. Then there is an $\varepsilon>0$ and a sequence $\left(x_{n}\right)$ in $B_{E}$ such that $\left|l_{n}\left(x_{n}\right)\right|>\varepsilon$ for each $n$. Let $x$ be in the $\sigma\left(E, E^{\prime}\right)$-closure of $\left\{x_{n}: n \in N\right\}$. In [9] Howard proved that a Banach space $E$ contains no subspace isomorphic to $l_{1}$ if and only if every bounded subset of $E$ is weakly sequentially dense in its weak closure. Therefore we can find a subsequence $\left(y_{n}\right)$ of $\left(x_{n}\right)$ such that $y_{n} \rightarrow x$ in $\sigma\left(E, E^{\prime}\right)$. Since $l_{n} \rightarrow 0$ in $\mu\left(E^{\prime}, E\right)_{s w}$ and thus also in $\sigma\left(E^{\prime}, E\right)$ there is an $n_{0} \in N$ such that $\sup _{k \in N}\left|l_{n}\left(y_{k}-x\right)\right| \leq \varepsilon / 2$ and $\left|l_{n}(x)\right| \leq \varepsilon / 2$ for $n \geq n_{0}$. Now, for $n \geq n_{0}, \varepsilon<\left|l_{n}\left(y_{n}\right)\right| \leq\left|l_{n}\left(y_{n}-x\right)\right|+\left|l_{n}(x)\right| \leq \varepsilon$, which is a contradiction. 
Combining Corollary 8 and Lemma 9 we arrive at the following corollary, which is valid for limited sets by Emmanuele [7].

Corollary 10. Let $E$ be a Banach space that contains no subspace isomorphic to $l_{1}$. Then every $C^{\infty}$-bounding subset of $\beta\left(E^{\prime}, E\right)$ is relatively compact in $\beta\left(E^{\prime}, E\right)$.

LEMMA 11. Let $C(T)$ be the Banach space of continuous functions on a compact, sequentially compact Hausdorff space $T$ with the supremum norm topology. Then $C(T)_{s w}$ and $C(T)$ have the same convergent sequences.

Proof. Assume that $f_{n} \rightarrow 0$ in $C(T)_{s w}$ but not in $C(T)$. Then there is an $\varepsilon>0$ and a sequence $\left(t_{n}\right)$ in $T$ such that $\left|f_{n}\left(t_{n}\right)\right|>\varepsilon$ for each $n$. Since $T$ is sequentially compact, we can find a convergent subsequence $\left(t_{n_{k}}\right)$ of $\left(t_{n}\right)$ which converges to $t \in T$. Now $T$ can be considered as a subspace of $C(T)_{c o}^{\prime}$ and therefore $t_{n_{k}} \rightarrow t$ in $\sigma\left(C(T)^{\prime}, C(T)\right)$ and $\left(t_{n_{k}}-t\right)$ is equicontinuous in $C(T)^{\prime}$. Since $f_{n} \rightarrow 0$ in $C(T)_{s w}$, there exists an $n_{0} \in N$ such that $\left|f_{n_{k}}\left(t_{n_{k}}\right)-f_{n_{k}}(t)\right| \leq \varepsilon / 2$ and $\left|f_{n_{k}}(t)\right| \leq \varepsilon / 2$ for $n \geq n_{0}$. As in the previous lemma we thus arrive at a contradiction.

By Diestel [5, p. 238], the next corollary also works for limited sets.

Corollary 12. Let $E$ be a Banach space which is isomorphic to a subspace of $C(T)$ with the supremum norm topology, where $T$ is a compact, sequentially compact Hausdorff space. Then every $C^{\infty}$-bounding subset of $E$ is relatively compact in $E$.

Recall that a Banach space $E$ is called weakly compactly generated (WCG), if $E$ is the closed linear span of some weakly compact set. All separable and reflexive Banach spaces as well as the space $c_{0}(\Gamma)$ are WCG spaces. For a WCG space $E$ the closed unit ball of the dual $E^{\prime}$ is $\sigma\left(E^{\prime}, E\right)$-sequentially compact. Therefore the classes of $C^{\infty}$-bounding and relatively compact sets coincide in WCG spaces.

In every infinite-dimensional Banach space $E$ there exist bounded non$C^{\infty}$-bounding sets. Indeed, let $B$ be the unit ball of $E$ and assume that $B$ is $C^{\infty}$-bounding. By Theorem $4, B$ is relatively $\sigma\left(E, E^{\prime}\right)$-compact and therefore $E$ is reflexive. But then $B$ is even relatively compact in $E$, by Corollary 12 , forcing $E$ to be finite-dimensional. It should be pointed out that this observation also could be obtained from the Josefson-Nissenzweig theorem [5]. 
COROLlary 13. Let $E$ be a quasi-complete lcs that is isomorphic to a subspace of a product $\prod_{i \in I} E_{l}$, where each $E_{1}$ is a WCG Banach space. Then every $C^{\infty}$-bounding subset of $E$ is relatively compact in $E$.

Proof. Let $A \subset E$ be $C^{\infty}$-bounding. Then $p r_{l}(A) \subset E_{l}$ is $C^{\infty}$-bounding and hence relatively compact for each $\imath \in I$, since $E_{l}$ is WCG. Therefore $A$ is relatively compact in $\prod_{t \in I} E_{t}$ and thus also in $E$.

A lcs is said to be transseparable, if it is a subspace of a topological product of separable normed spaces [14]. Since the completion of a separable normed space is separable, every $C^{\infty}$-bounding subset of a quasi-complete transseparable lcs is relatively compact. According to [14], a lcs $E$ is transseparable if and only if for every zero-neighbourhood $U$ in $E$ there is a countable subset $M$ of $E$ with $E=M+U$. Thus the class of transseparable spaces contains not only the semi-weak ones but all separable and Lindelöf locally convex spaces as well. For Lindelöf spaces, however, we can characterize the $C^{\infty}$-bounding sets without quasi-completeness by using the interpolation result below.

Proposition 14. Let $E$ be a Lindelöf lcs. Assume that $\left(x_{n}\right)$ is a sequence of distinct elements in $E$ without cluster points. Then there exists a function $f \in C^{\infty}(E)$ such that $f\left(x_{n}\right)=n$ for each $n$.

Proof. As the sequence $\left(x_{n}\right)$ has no cluster points in $E$, there is a convex open neighbourhood $A_{n}$ of each $x_{n}$ such that $x_{k} \notin \overline{A_{n}}$ for $k \neq n$. By the same argument there exists a convex open neighbourhood $B_{x}$ of every $x$ outside the sequence $\left(x_{n}\right)$ such that $\bar{B}_{x} \cap\left\{x_{n}: n \in N\right\}=\varnothing$. Since $E$ is Lindelöf, there is a sequence $\left(B_{k}\right)$ of convex open sets with $E=\bigcup_{n=1}^{\infty} A_{n} \cup B_{n}$ and $\overline{B_{k}} \cap\left\{x_{n}: n \in N\right\}=\varnothing$ for each $k$. Let $W_{n}=\bigcup_{i=1}^{n} A_{i} \cup B_{i}$. Hence $\left(W_{n}\right)$ is an increasing open covering of $E$, where the closures $\bar{W}_{n}$ are $\sigma\left(E, E^{\prime}\right)$ closed sets with $x_{k} \notin \overline{W_{n}}$ for $k>n$. Let $f_{1}$ be the constant function with the value 1 . Since the weak topology is $C^{\infty}$-smooth, we can find a sequence $\left(f_{n}\right)$ of functions in $C^{\infty}(E)$ such that

$$
f_{n}\left(\overline{W_{n-1}}\right)=\{0\} \quad \text { and } f_{n}\left(x_{n}\right)=n-1-\sum_{i=2}^{n-1} f_{i}\left(x_{n}\right), n=2,3, \ldots
$$

Let $f(x)=\sum_{n=1}^{\infty} f_{n}(x)$. By construction, $f$ is locally finite and thus a function in $C^{\infty}(E)$. Furthermore $f\left(x_{n}\right)=n$ for each $n$. 
Since the compact and countably compact sets are the same in Lindelöf spaces we arrive at

COROLlaRY 15. Every $C^{\infty}$-bounding set in a Lindelöf lcs is relatively compact.

\title{
References
}

[1] C. Aliprantis and O. Burkinshaw, Positive Operators (Academic Press, San Diego, 1985).

[2] P. Biström, S. Bjon, and M. Lindström, 'Function algebras on which homomorphisms are point evaluations on sequences', Manuscripta Math. 73 (1991), 179-185.

[3] J. Bourgain and J. Diestel, 'Limited operators and strict cosingularity', Math. Nachr. 119 (1984), 55-58.

[4] J. Dazord, 'Factoring operators through $c_{0}$ ', Math. Ann. 220 (1976), 105-122.

[5] J. Diestel, Sequences and Series in Banach spaces, Graduate Texts in Math. 97 (Springer, Berlin, 1984).

[6] S. Dineen, 'Unbounded holomorphic functions on a Banach space', J. London Math. Soc. 4 (1972), 461-465.

[7] G. Emmanuele, 'A dual characterization of Banach spaces not containing $l_{1}$ ', Bull. Acad. Polon. Sci. Sér. Sci. Math. 34 (1986), 155-159.

[8] K. Floret, Weakly compact sets, Lecture Notes in Math. 801 (Springer, Berlin, 1980).

[9] J. Howard, 'Weak sequential denseness in Banach spaces', Proc. Amer. Math. Soc. 99 (1987), 351-352 and 104, 334 (1988).

[10] H. Jarchow, Locally convex spaces (Teubner, Stuttgard, 1981).

[11] A. Kriegl and L. Nel, 'Convenient vector spaces of smooth functions', Math. Nachr. 147 (1990), 39-45.

[12] M. Lindström and Th. Schlumprecht, 'On limitedness in locally convex spaces', Arch. Math. (Basel) 53 (1989), 65-74.

[13] J. Lloyd, 'Smooth partitions of unity on manifolds', Trans. Amer. Math. Soc. 197 (1974), 249-259.

[14] P. Pérez Carreras and J. Bonet, Barrelled locally convex spaces (North-Holland, Amsterdam, 1987).

[15] M. Valdivia, 'Some new results on weak compactness', J. Funct. Anal. 24 (1977), 1-10.

\author{
Åbo Akademi \\ SF-20500 Ảbo \\ Finland
}

